\title{
A review of juice-based oral nutritional supplements in inpatients: patient satisfaction and nutritional composition
}

\author{
A. Durr, C. T. McArdle, S. McGaughey, J. Homes, A. Cavanagh and L. Moorhead \\ Department of Nutrition and Dietetics, Royal Victoria Hospital, Belfast Health and Social Services Trust, Belfast BT12 6BA
}

\begin{abstract}
Malnutrition in the UK is estimated to cost the NHS $£ 7.3$ billion per year which is more than double the bill for obesity ${ }^{(1)}$. Oral nutritional supplements (ONS) have shown clinical improvements in malnourished patients. Juice-based ONS are a useful means of providing supplementary energy and protein but they are not nutritionally complete. Enlive plus is the standard juice-based ONS contracted for use within the Royal Victoria Hospital. There is an average estimated use of 6890 cartons per year. The aim of this audit was to evaluate patient satisfaction and nutritional composition with the current standard juice-based drink and other juice-based ONS currently approved by the advisory committee on borderline substances (ACBS).

Patients meeting selected criteria and providing informed consent were included in the audit. All sweet flavours $(n$ 8) of current ONS supplements were tested. Flavour combinations were presented to patients in a single blinded manner and a questionnaire was completed. Each patient scored attributes for each ONS flavour sampled. Attributes included smell, colour, taste, consistency, after-taste and overall acceptability. Total scores were collected and weighted for taste. The juice-based ONS were also compared for nutritional content and differences in packaging, ease of opening and other differences were evaluated.

A total of 115 patients participated in the audit, fifty-four were male and sixty-one female. The mean age was 57, (range 16-93) years. The most popular ONS were Enlive plus, which scored $66 \%$ overall and Fortijuce scoring $65 \%$. Enlive plus scored $78 \%$ for colour compared to Fortijuce which scored $63 \%$. This maybe due to the presence of E numbers contained in Enlive plus. When colour was excluded from the analysis Fortijuce was the most preferred drink in our patient sample (66\%). The Table shows the most preferred drink flavour in relation to total score. The most popular drink flavour in relation to taste and total score was Fortijuce apple flavour (84\%). The energy and protein content are similar in both the Enlive plus and Fortijuce while, the sugar content is lower in Enlive plus.
\end{abstract}

\begin{tabular}{lc}
\hline Supplement drink & $\begin{array}{c}\text { Score } \\
(\%)\end{array}$ \\
\hline Fortijuce, apple & 84 \\
Enlive plus, peach & 80 \\
Enlive plus, strawberry & 68 \\
Fortijuce, strawberry & 67 \\
Fortijuce, tropical & 65 \\
Fortijuce, forest fruits & 63 \\
Provide Xtra, orange \& pineapple & 62 \\
Enlive, lemon \& lime & 59 \\
Clinutren, pear \& cherry & 59 \\
\hline
\end{tabular}

The most popular ONS were Fortijuce and Enlive plus with only a $1 \%$ difference in their total score. The current contracted flavours in the Royal Victoria Hospital are Enlive lemon \& lime, orange and fruit punch; however, the most preferred overall flavours for Enlive were found to be peach, strawberry for Fortijuce, apple, strawberry and forest fruit. These results will be used to inform the procurement process for juice-based ONS in the hospital. A change in the flavours of current ONS available for patients may improve patient satisfaction.

1. Bapen UK (2005) The cost of disease-related malnutrition in the UK and economic considerations for the use of oral nutritional supplements (ONS) in adults. 